\title{
ASSESSING DRIVING PERFORMANCE WITH MODERATE VISUAL FIELD LOSS
}

\author{
Alex Bowers, Eli Peli \\ The Schepens Eye Research Institute \\ Harvard Medical School \\ Boston, Massachusetts, USA \\ E-mail: abowers@vision.eri.harvard.edu \\ Jennifer Elgin ${ }^{1}$, Gerald McGwin Jr. ${ }^{1,2,3}$,Cynthia Owsley ${ }^{1}$ \\ ${ }^{1}$ Department of Ophthalmology, School of Medicine \\ ${ }^{2}$ Department of Epidemiology and International Health, School of Public Health \\ ${ }^{3}$ Department of Surgery, School of Medicine \\ University of Alabama at Birmingham \\ Birmingham, Alabama, USA
}

\begin{abstract}
Summary: The minimum binocular horizontal field extent for driver licensing varies widely between states in the USA. We examined the relationship between visual field extent and open-road driving performance using a scoring method that measured the quality of specific skills for a range of general driving maneuvers, as well as maneuvers that we expected to be difficult for people with restricted fields. Twenty-eight current drivers with mild to moderate peripheral visual field restrictions $\left(123 \pm 20^{\circ}\right.$, V4e target $)$ drove the 14-mile route. While most subjects were scored as safe drivers, those with more restricted horizontal and vertical binocular field extents showed significantly poorer skills in maneuvers for which a wide field of vision is likely to be important ( $p \leq 0.05$ ): speed matching when changing lanes, and maintaining lane position and keeping to the path of the curve when driving around curves. Further studies using similar assessment methods with drivers with more restricted fields are necessary to determine the minimum field extent for safe driving.
\end{abstract}

\section{INTRODUCTION}

It is unclear what minimum size of the visual field is consistent with safe driving; only 31 jurisdictions in the USA specify a minimum binocular horizontal field extent for driver licensing, ranging from $20^{\circ}$ to $150^{\circ}$ (Peli \& Peli, 2002). Although Johnson and Keltner (1983) found a doubling of crashes and traffic violations in people with binocular peripheral visual field loss, other studies have found no significant association between crash rate and visual field deficits (Ball et al., 1993; Burg, 1967; Danielson, 1957; Decina \& Staplin, 1993; Owsley et al., 1998). Because crashes are rare events, large samples are needed to address questions about visual field extent and driver safety; however, the study of driving performance is more amenable to smaller sample studies. On-road closed-course assessments of driving with simulated binocular field restrictions (Wood et al., 1993; Wood \& Troutbeck, 1992, 1994, 1995) and driving simulator assessments of patients with real visual field loss (Coeckelbergh et al., 2002; Szlyk et al., 1992), suggest a relationship between binocular field extent and driving performance even for moderate field loss; however, these approaches do not evaluate the real-world conditions of on-road driving. 
Here we describe an exploratory study of the relationship between visual field extent and driving skills in an open, on-road environment. A scoring method was developed (Sodhi et al., in press) that measured the quality of specific skills and maneuvers that were a priori expected to be negatively impacted by peripheral field loss (e.g., lane position and path keeping during curve taking or gap judgment when changing lanes or turning at an intersection), as well as skills and maneuvers that we expected would not be affected by peripheral field loss (e.g., stopping distance at an intersection or speed appropriateness on a straight road segment).

\section{METHODS}

\section{Subjects}

Twenty-eight patients seen in ophthalmology clinics, affiliated to the University of Alabama at Birmingham (UAB), in the Callahan Eye Foundation Hospital over the previous 12-month period who had conditions that could cause peripheral visual field loss (e.g., glaucoma, retinitis pigmentosa) participated in the study. All subjects held a current driver's license in Alabama and still drove. The Institutional Review Boards of UAB and Schepens Eye Research Institute approved the study. All subjects signed an informed consent form after the nature and purpose of the study were explained.

\section{Procedures}

The study visit (2-3 hours) took place in the Driving Assessment Clinic at UAB. In-clinic functional assessments including vision measures, general health questionnaire, driving habits questionnaire (Owsley et al., 1999) and the mini-mental status examination (MMSE) (Folstein et al., 1975) were carried out. Visual acuity for distance was measured with habitual (walk-in) correction using the Early Treatment for Diabetic Retinopathy Study chart (Ferris et al., 1982) and its standard protocol, and expressed as logMAR. The extent of the monocular visual field in each eye was plotted with the Goldmann perimeter using the V4e target in a combined static /dynamic procedure. The two monocular field plots were used to derive the extent of the binocular field. Three binocular field scores were determined: the horizontal extent along the $0^{\circ}$ $180^{\circ}$ meridian (maximum extent $180^{\circ}$ ), typically used when specifying the field extent for driver licensing; the vertical extent along the $90^{\circ}-270^{\circ}$ meridian (maximum extent about $130^{\circ}$ ); and a measure of the overall field extent, the sum of the extents along 12 meridians separated by $30^{\circ}$ (maximum extent about $970^{\circ}$ ).

Following completion of the in-clinic functional assessment, the on-road evaluation was conducted. Assessments took place between 10.00 A.M. and 3.30 P.M. to avoid rush hour traffic conditions, in both sunny and cloudy weather conditions (but not when it was raining, snowing or foggy). The course included a representative variety of general driving maneuvers, as well as maneuvers expected to be difficult for people with peripheral visual field loss. The total length of the course was 14 miles on roads in Birmingham, AL. All subjects drove the same course. Two evaluators scored driving performance: a certified driving rehabilitation specialist (CDRS) in the front passenger seat, with a second trained evaluator in the back seat of the vehicle (a 1998 Chevrolet Lumina equipped with a passenger side brake). 
The course was divided into two sections: a preliminary off-road section, followed by an on-road section. In the preliminary section, vehicle control skills were evaluated in a parking lot to ensure that the participant had adequate control of the unfamiliar vehicle before proceeding to on-road driving. The on-road section started on low-traffic roads and then proceeded to busier roads and interstate driving. The route comprised a variety of road types, intersections, and sections with curbed and uncurbed edges, straight sections and curves. At pre-determined points along the route, performance of specific maneuvers (turning right and left at intersections, merging, changing lanes to right and left, crossing intersections, lane control on a straight road and curve taking on right and left curves) was scored with respect to the following itemized driving skills: lane position (before and after, or during the maneuver), gap judgment, steering steadiness, spacing, appropriateness of speed for the maneuver, ability to adjust or match speed to traffic in a new lane, path keeping when going around a curve, and stop position relative to the end of the lane at an intersection. The back-seat evaluator, masked to the visual field characteristics of the participant driver, carried out this scoring, using a separate score sheet for each maneuver. A 5point scale was used: 1, driving evaluator had to take control of car; 2, skill performed in an unsafe manner (but evaluator did not have to take control); 3 skill performed in an unsatisfactory manner (but did not compromise safety); 4, skill performed in a satisfactory manner; 5 , skill performed in a flawless manner.

Global driving performance for the entire drive was assessed at the end of the on-road test by both the front-seat CDRS and the back-seat evaluator. Ratings were made on a 5-point scale (as above) on the following items: interaction with other traffic, anticipatory skills (margin of error), vehicle control, reaction to unexpected events, adjustment of speed to traffic conditions, and overall driving. Global driving was also assessed for interstate driving in terms of the first four items; as the CDRS focused on safe control of the vehicle during interstate travel, only the backseat evaluator made ratings of interstate driving.

\section{Statistical Analysis}

Spearman correlation coefficients and associated tests of significance were calculated between ratings of driving performance (driving skills for each maneuver and global scores) and the three binocular Goldmann visual field extent measures. Partial correlation coefficients adjusting for the potential confounding factors of age and cognitive status score were also calculated. A pvalue of $\leq 0.05$ was considered statistically significant.

\section{RESULTS}

The majority of the 28 participants were older adults (mean $67 \pm 14$, range 33 to 84 years), male (64\%), and split about evenly between African Americans (46\%) and whites (50\%). The etiology of vision impairment was almost entirely due to primary open angle glaucoma (27 of 28), with one participant having retinitis pigmentosa. Participants averaged approximately 2 to 3 comorbid medical conditions, and cognitive status was good (MMSE score $28 \pm 2$ ). Participants reported driving on average 5 to 6 days per week, with typical weekly mileages varying from 2 to 348 (median 126 miles), compared with an average of 200 miles per week for adults of all ages in the USA and 120 miles per week for those aged over 65 (U.S. Department of Transportation, 2003). All subjects were drivers since young adulthood. Binocular visual acuity was good, averaging 20/25 (0.09 $\pm 0.11 \log$ MAR) and binocular visual field loss was 
(qualitatively) mild to moderate. The binocular horizontal field extent ranged from $78^{\circ}$ to $165^{\circ}$ (mean $123 \pm 20^{\circ}$ ), the binocular vertical field extent from $50^{\circ}$ to $124^{\circ}$ (mean $89 \pm 21^{\circ}$ ) and the binocular total field extent from $412^{\circ}$ to $836^{\circ}$ (mean $628 \pm 117^{\circ}$ ). Eighty percent would have met the requirement for driver licensing in 16 states in the USA with minimum horizontal field diameters of $20^{\circ}$ to $110^{\circ}$ (in addition to qualifying in the 18 jurisdictions without visual field requirements). The correlations between each of the maneuver/skill combinations and the binocular visual field variables, adjusted for age and cognitive status, are summarized in Table 1.

Table 1. Spearman's correlations (p-values) between ratings on specific driving maneuvers and binocular visual field scores, adjusted for age and cognitive status

\begin{tabular}{|c|c|c|c|c|}
\hline $\begin{array}{l}\text { Driving Maneuver (number assessed) }{ }^{1} \\
\text { Skills scored }\end{array}$ & $\mathrm{N}^{2}$ & $\begin{array}{c}\text { Binocular } \\
\text { Horizontal Field }\end{array}$ & $\begin{array}{c}\text { Binocular } \\
\text { Vertical Field }\end{array}$ & $\begin{array}{l}\text { Binocular } \\
\text { Total Field }\end{array}$ \\
\hline \multicolumn{5}{|l|}{ Turning ( 3 left and 3 right) } \\
\hline Lane position before and after & 28 & $0.123(0.548)$ & $0.280(0.166)$ & $0.211(0.301)$ \\
\hline Gap judgment & 28 & $0.052(0.802)$ & $0.192(0.346)$ & $0.027(0.898)$ \\
\hline Path keeping & 28 & $0.099(0.632)$ & $0.419(0.033) *$ & $0.299(0.137)$ \\
\hline Speed appropriate & 28 & $0.214(0.294)$ & $0.174(0.394)$ & $0.288(0.153)$ \\
\hline Stop position & 28 & $0.143(0.485)$ & $0.207(0.310)$ & $0.146(0.477)$ \\
\hline \multicolumn{5}{|l|}{ Changing lanes ( 3 left and 3 right) } \\
\hline Lane position before and after & 27 & $0.379(0.062)$ & $0.281(0.173)$ & $0.330(0.108)$ \\
\hline Gap judgment & 27 & $0.106(0.615)$ & $0.310(0.132)$ & $0.348(0.088)$ \\
\hline Steering steadiness & 27 & $0.166(0.427)$ & $0.184(0.378)$ & $0.161(0.442)$ \\
\hline Speed match & 27 & $0.555(0.004)^{*}$ & $0.587(0.002)^{*}$ & $0.472(0.017)^{*}$ \\
\hline \multicolumn{5}{|l|}{ Crossing intersection (3) } \\
\hline Lane position before and after & 28 & $0.170(0.405)$ & $0.151(0.461)$ & $0.085(0.678)$ \\
\hline Steering steadiness & 28 & $0.231(0.257)$ & $0.165(0.421)$ & $0.167(0.415)$ \\
\hline Speed appropriate & 28 & $0.309(0.125)$ & $0.291(0.149)$ & $0.340(0.090)$ \\
\hline Stop position & 28 & $0.194(0.342)$ & $0.381(0.055)$ & $0.104(0.614)$ \\
\hline \multicolumn{5}{|l|}{ Straight road (1) } \\
\hline Lane position during maneuver & 27 & $0.071(0.736)$ & $0.226(0.277)$ & $0.196(0.347)$ \\
\hline Steering steadiness & 27 & $-0.050(0.813)$ & $0.169(0.420)$ & $0.121(0.564)$ \\
\hline Speed appropriate & 27 & $0.301(0.144)$ & $0.306(0.138)$ & $0.177(0.397)$ \\
\hline Spacing & 27 & $0.351(0.086)$ & $0.327(0.110)$ & $0.267(0.197)$ \\
\hline \multicolumn{5}{|l|}{ Curve taking ( 2 left and 2 right $)$} \\
\hline Lane position during maneuver & 28 & $0.509(0.008)^{*}$ & $0.547(0.004)^{*}$ & $0.567(0.003) *$ \\
\hline Path keeping & 28 & $0.460(0.018)^{*}$ & $0.580(0.002)^{*}$ & $0.577(0.002)^{*}$ \\
\hline Steering steadiness & 28 & $0.232(0.254)$ & $0.277(0.171)$ & $0.289(0.153)$ \\
\hline Speed appropriate & 28 & $0.375(0.059)$ & $0.318(0.114)$ & $0.311(0.122)$ \\
\hline Spacing & 22 & $0.445(0.049) *$ & $0.343(0.139)$ & $0.446(0.049) *$ \\
\hline
\end{tabular}

${ }^{1}$ For driving maneuvers that were assessed more than once, the mean score for each driving skill was computed and used in correlation analyses

${ }^{2} \mathrm{~N}$ is not always 28 , as some measures could not be scored or were not applicable for all participants.

$* \mathrm{p} \leq 0.05$

A more restricted binocular horizontal field, vertical field and total field were all significantly $(\mathrm{p} \leq 0.05)$ associated with poorer performance in speed matching when changing lanes, and with 
poorer performance in path keeping (Figure 1a) and lane positioning during curve taking, as expected. In addition, both a restricted horizontal and a restricted total field were significantly associated with poorer performance in maintaining an appropriate following distance (spacing) during curve taking, and a more restricted vertical field was related to poorer performance in path keeping when turning.

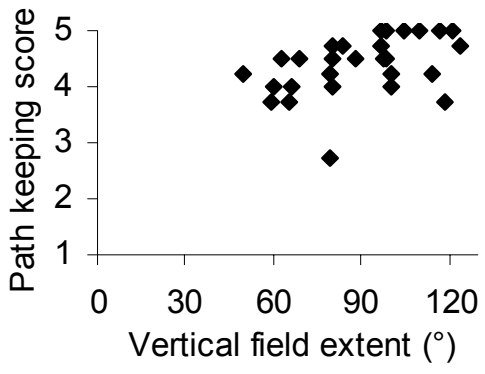

(a)

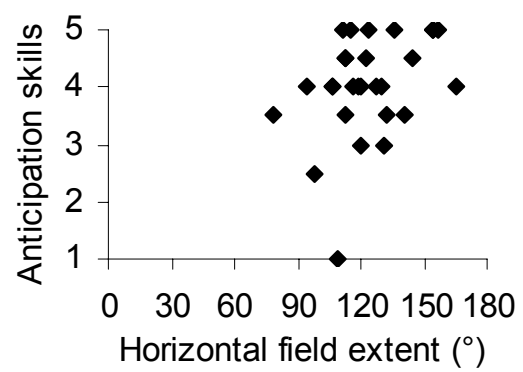

(b)

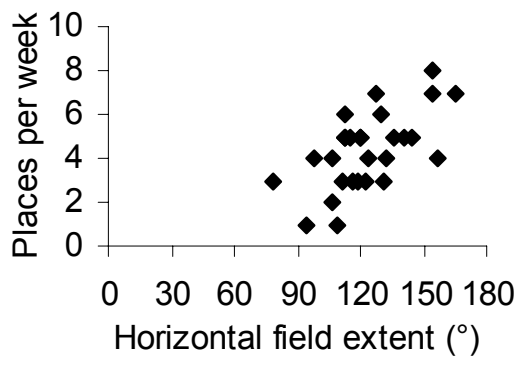

(c)

Figure 1. Scatter plots of raw unadjusted data for which the correlations (adjusted for age and cognitive status) were significant ( $p \leq 0.05)$ : (a) A more restricted vertical field was associated with poorer path-keeping during curve-taking, (b) A more restricted horizontal field was associated with poorer ratings for anticipation skills, (c) Drivers with more restricted horizontal fields self-limited their driving and drove to fewer places.

The correlations between each of the global ratings and the binocular visual field variables, adjusted for age and cognitive status, are summarized in Table 2 . A reduced width of the horizontal field, a restricted vertical field and a reduced total field were all significantly $(p \leq 0.05)$ related to poorer ratings for anticipatory skills (Figure $2 b$ ) and interaction with other traffic on the interstate. In addition, a restricted vertical field was significantly associated with difficulties adjusting speed to traffic conditions and with poorer scores on reaction to the unexpected in the roadway during interstate driving.

\section{DISCUSSION}

The results of this study suggest that even mild to moderate peripheral visual field restrictions can negatively impact specific driving skills, especially for maneuvers for which a wide field of vision may be important. Drivers with more restricted fields showed poorer skills in speed matching when changing lanes, and poorer skills in maintaining lane position and keeping to the path of the curve when driving around curves. By comparison, there were no significant correlations between field extent and driving skills in other situations that require information primarily from the central visual field - e.g., maintaining appropriate following distance and speed on straight road segments. The effects of restricted peripheral fields on driving skills were also apparent in the summary measures of driving performance, assessed for the entire test drive, where more restricted fields were associated with poorer ratings for anticipatory skills. Currently, visual field requirements for driver licensing are primarily specified in terms of the horizontal field diameter (Peli \& Peli, 2002). However, our results suggest that vertical field extent may also be an important consideration, especially in turning or curve-taking maneuvers. 
Table 2. Spearman's correlations (p-values) between summary driving ratings and binocular visual field scores, adjusted for age and cognitive status

\begin{tabular}{|c|c|c|c|c|}
\hline & $\mathbf{N}^{1}$ & $\begin{array}{c}\text { Binocular } \\
\text { Horizontal Field }\end{array}$ & $\begin{array}{c}\text { Binocular } \\
\text { Vertical Field }\end{array}$ & $\begin{array}{l}\text { Binocular } \\
\text { Total Field }\end{array}$ \\
\hline \multicolumn{5}{|l|}{ Entire drive $^{2}$} \\
\hline Interaction with other traffic & 28 & $0.350(0.080)$ & $0.339(0.090)$ & $0.324(0.107)$ \\
\hline Anticipatory skills & 28 & $0.462(0.018) *$ & $0.506(0.008)^{*}$ & $0.456(0.019) *$ \\
\hline Vehicle control skills & 28 & $0.153(0.454)$ & $0.365(0.067)$ & $0.255(0.209)$ \\
\hline Adjusting speed to conditions & 24 & $0.400(0.067)$ & $0.508(0.016)^{*}$ & $0.300(0.175)$ \\
\hline Reaction to unexpected & 18 & $0.488(0.055)$ & $0.331(0.210)$ & $0.314(0.236)$ \\
\hline Overall driving performance & 28 & $0.320(0.111)$ & $0.274(0.176)$ & $0.264(0.193)$ \\
\hline \multicolumn{5}{|l|}{ Interstate $^{3}$} \\
\hline Interaction with other traffic & 24 & $0.418(0.053)^{*}$ & $0.479(0.024)^{*}$ & $0.487(0.022)^{*}$ \\
\hline Anticipatory skills & 24 & $0.339(0.123)$ & $0.309(0.162)$ & $0.342(0.119)$ \\
\hline Vehicle control skills & 23 & $0.018(0.939)$ & $0.306(0.177)$ & $0.233(0.311)$ \\
\hline Reaction to unexpected & 17 & $0.308(0.265)$ & $0.517(0.049)^{*}$ & $0.434(0.106)$ \\
\hline
\end{tabular}

${ }^{1} \mathrm{~N}$ is not always 28 , as some measures could not be scored (e.g. if no unexpected events occurred during a drive) or were not applicable for all participants (four would not drive on the interstate).

2 Mean of two raters' scores

3 Scored by back-seat evaluator only

$* \mathrm{p} \leq 0.05$

Previous studies, which have reported an effect of peripheral visual field restriction on driving performance, have either used simulations of severe visual field restrictions on a closed course (Wood et al., 1993; Wood \& Troutbeck, 1992, 1995), or have carried out driving simulator evaluations using patients with more restricted fields than the sample in this study (Coeckelbergh et al., 2002; Szlyk et al., 1992). The only study with a similar range of mild to moderately restricted horizontal field extents $\left(130^{\circ} \pm 21^{\circ}\right.$, range $\left.70^{\circ}-140^{\circ}\right)$ (Szlyk et al., 2002) failed to find any significant correlations between binocular field width and driving performance in a driving simulator. The difference in findings between this driving simulator evaluation and our on-road evaluation of drivers with mild to moderate peripheral field loss may relate to differences in the duration of the driving assessment ( 5 minutes compared to $>30$ minutes), differences in the driving environment (virtual compared to a real traffic environment), or differences in the sensitivity of the assessment to the effects of peripheral field loss (scoring using a general set of performance indices compared to detailed scoring of specific skills and maneuvers).

The majority of driving skill ratings in our study were in the 3 to 5 range, i.e., from unsatisfactory (but does not compromise safety) through to excellent; only 1 participant was rated unsafe to drive based on driving performance across the whole course (his horizontal visual field was $109^{\circ}$ ). By comparison, in an on-road study of drivers with more restricted peripheral fields than the range in this study (mean $84^{\circ} \pm 35^{\circ}$ compared to $123^{\circ} \pm 20^{\circ}$ here), only $43 \%$ passed a test of practical fitness to drive (Coeckelbergh et al., 2002). As previously reported for drivers with a diagnosis of glaucoma (McGwin et al., 2004), drivers in the current study appeared to be aware of their visual limitations and self-regulated their driving accordingly; those with more restricted fields drove to fewer places (Figure 1c). 
Our results demonstrate, in a small sample of drivers, that mild to moderate peripheral visual field restrictions adversely affect specific driving skills in maneuvers for which a wide field of vision is likely to be important (although the majority of subjects were regarded as safe drivers). From this preliminary study, the skills and maneuvers, which should be evaluated in subsequent investigations of driving with peripheral field loss, can be identified. A follow-up study employing similar assessment methods with a larger sample of drivers and a wider range of field extents would be necessary to determine the minimum field extent required for safe driving.

\section{ACKNOWLEDGMENTS}

This research was supported by NIH grants EY12860 (EP), P50-AG11684 (CO) and R21EY14071 (CO), Research to Prevent Blindness, Inc., the EyeSight Foundation of Alabama, and the UAB Driving Assessment Clinic. Cynthia Owsley is a Research to Prevent Blindness Senior Scientific Investigator. We thank Mark Tant and Kent Higgins for help in the conceptual design of driving test courses and assessment procedures for the visually impaired.

\section{REFERENCES}

Ball, K., Owsley, C., Sloane, M. E., Roenker, D. L., \& Bruni, J. R. (1993). Visual attention problems as a predictor of vehicle crashes in older drivers. Investigative Ophthalmology and Visual Science, 34, 3110-3123.

Burg, A. (1967). The relationship between vision test scores and driving record: General findings, Report 67-24. Los Angeles: University of California, Los Angeles, Department of Engineering.

Coeckelbergh, T. R., Brouwer, W. H., Cornelissen, F. W., van Wolffelaar, P., \& Kooijman, A. C. (2002). The effect of visual field defects on driving performance: a driving simulator study. Archives of Ophthalmology, 120, 1509-1516.

Danielson, R. W. (1957). The relationship of fields of vision to safety in driving, with a report of 680 drivers examined by various screening methods. American Journal of Ophthalmology, 44(4), 657-680.

Decina, L. E., \& Staplin, L. (1993). Retrospective evaluation of alternative vision screening criteria for older and younger drivers. Accident Analysis and Prevention, 25, 267-275.

Ferris, F. L., III, Kassoff, A., Bresnick, G. H., \& Bailey, I. (1982). New visual acuity charts for clinical research. American Journal of Ophthalmology, 94, 91-96.

Folstein, M. F., Folstein, S. E., \& McHugh, P. R. (1975). "Mini-mental state": a practical method for grading the cognitive state of patients for the clinician. Journal of Psychiatric Research, 12, 189-198.

Johnson, C. A., \& Keltner, J. L. (1983). Incidence of visual field loss in 20,000 eyes and its relationship to driving performance. Archives of Ophthalmology, 101, 371-375.

McGwin, G., Jr, Mays, A., Joiner, W., DeCarlo, D. K., McNeal, S., \& Owsley, C. (2004). Is glaucoma associated with motor vehicle collision involvement and driving avoidance? Investigative Ophthalmology and Visual Science, 45, 3934-3939. 
Owsley, C., Ball, K., McGwin, G., Jr, Sloane, M. E., Roenker, D. L., White, M. F., \& Overly, E. T. (1998). Visual processing impairment and risk of motor vehicle crash among older adults. Journal of the American Medical Association, 279, 1083-1088.

Owsley, C., Stalvey, B., Wells, J., \& Sloane, M. E. (1999). Older drivers and cataract: Driving habits and crash risk. Journal of Gerontology: Medical Sciences, 54A, M203-M211.

Peli, E., \& Peli, D. (2002). Driving with confidence: A practical guide to driving with low vision. River Edge, New Jersey: World Scientific Press.

Sodhi, M., Reimer, B., Tant, M., Peli, E., Bowers, A. R., Woods, R. L., Higgins, K., \& Turco, P. (in press). Driver performance evaluation: Considerations underlying selection and design of routes, Vision in Vehicles X. Amsterdam: Elsevier Sciences Publishers.

Szlyk, J. P., Alexander, K. R., Severing, K., \& Fishman, G. A. (1992). Assessment of driving performance in patients with retinitis pigmentosa. Archives of Ophthalmology, 110, 17091713.

Szlyk, J. P., Taglia, D. P., Paliga, J., Edward, D. P., \& Wilensky, J. T. (2002). Driving performance in patients with mild to moderate glaucomatous clinical vision changes. Journal of Rehabilitation Research and Development, 39(4), 467-495.

U.S. Department of Transportation. (2003). NHTS 2001 Highlights Report, BTS03-05. Washington, DC: Bureau of Transportation Statistics.

Wood, J. M., Dique, T., \& Troutbeck, R. (1993). The effect of artificial visual impairment on functional visual fields and driving performance. Clinical Vision Science, 8, 563-575.

Wood, J. M., \& Troutbeck, R. (1992). Effect of restriction of the binocular visual field on driving performance. Ophthalmic and Physiological Optics, 12, 291-298.

Wood, J. M., \& Troutbeck, R. (1994). Effect of visual impairment on driving. Human Factors, $36,476-487$.

Wood, J. M., \& Troutbeck, R. (1995). Elderly drivers and simulated visual impairment. Optometry and Vision Science, 72, 115-124. 